\title{
Chronic Pancreatitis and Pancreatic Cancer
}

\author{
Xiangyu Kong Tao Sun Fanyang Kong Yiqi Du Zhaoshen Li \\ Department of Gastroenterology, Shanghai Changhai Hospital, Second Military Medical \\ University, Shanghai, People's Republic of China
}

\section{Key Words}

Chronic pancreatitis · Macrophage · Microenvironment · Pancreatic cancer · Signaling pathways

\begin{abstract}
Background: Pancreatic cancer (PC) is one of the most lethal diseases with an incidence rate almost equal to the rate of mortality. Chronic pancreatitis (CP) is a common chronic inflammatory disease of unknown etiology that affects the pancreas. Epidemiological studies have identified CP to be a major risk factor for PC. Summary: A greater understanding of the molecular mechanisms linking $\mathrm{CP}$ and $\mathrm{PC}$ has identified several common pathways that provide targets for future interventions. This article reviews those components in the CP-PC connection, including the role of macrophages, the maintenance of genome stability, cytokines, and other nodal factors such as nuclear factor kappa B, COX-2 and reactive oxygen species. Key Message: The molecular mechanisms that underlie $\mathrm{CP}$ and $\mathrm{PC}$ provide novel targets for future therapies for PC. Practical Implications: The stromal-desmoplastic reaction plays an important role in initiating and sustaining chronic inflammation and tumor progression. Recently, two targeted anti-tumor agents, erlotinib and nab-paclitaxel, have shown promising therapeutic efficacy. Notably, both these agents target components (EGFR and SPARC) within the inflammatory stroma surrounding malignant cells, underscoring the importance of inflammation in pancreatic carcinogenesis. Identifying the common pathways linking CP and PC may help uncover additional novel targets for future therapies.




\section{Introduction}

A friend of mine with chronic pancreatitis (CP) visited me and said he had been annoyed by abdominal pain for more than 1 year. An abdominal CT scan showed that a tumor mass had developed in his pancreas; histopathology determined its malignant nature. I was shocked because though he had undergone 10 years of follow-up, pancreatic cancer (PC) eventually occurred. Epidemiological studies have shown that $\mathrm{CP}$ is one of the major risk factor for PC, with a 2.3- to 18.5-fold increased risk compared to normal controls. Delineating the progression from $\mathrm{CP}$ to $\mathrm{PC}$ and identifying the genetic events accompanying this progression will help us detect early-stage PC in CP cases and improve the prognosis of these patients.

Hereditary pancreatitis represents the best model to dissect the causal linkage between $\mathrm{CP}$ and $\mathrm{PC}$, and epidemiological analysis showed that hereditary pancreatitis ranks the strongest known risk factor for PC [1]. With the advent of molecular techniques, several germline mutations within certain genes (e.g. PRSS1 and CFTR) were identified as the major cause for hereditary pancreatitis. However, these mutations were rarely detected in common $\mathrm{CP}$ cases, which indicates that these genes are not directly associated with the development of PC. Now it is widely accepted that inflammation itself, but not those hereditary genes, promotes PC progression.

Once inflammation is initiated, inflammatory cells, especially those macrophages, are recruited to specific sites and release cytokines, growth factors and matrix-degrading enzymes. All these cytokines, in combination with macrophages, constitute the extrinsic signals and will teach the microenvironment to facilitate PC growth. Furthermore, the inflammatory milieu may help eradicate surveillance mechanisms and destabilize the genome in normal pancreatic cells, which will enhance accumulation of genetic events within these cells and accelerate the process of pancreatic tumor formation. A number of dysregulated signaling pathways have been commonly identified in both CP and PC tissues. Of note, most of these altered pathways, including cytokines, nuclear factor kappa B (NF- $\kappa \mathrm{B})$, reactive oxygen species (ROS), peroxisome proliferator-activated receptor- $\gamma$ (PPAR $\gamma$ ), etc. have been validated to hold therapeutic potential value in PC treatment.

$\mathrm{CP}$ and $\mathrm{PC}$ are two entirely different diseases with distinct prognoses. However, in certain cases it is quite difficult to distinguish among them. As too many altered signaling pathways occur in linkage between ' $\mathrm{CP}$ and $\mathrm{PC}$ ', it is a big challenge to identify markers with potential for differential diagnosis. Until now, none of the global proteomic studies have identified PC-specific makers, which may partially be attributed to the low abundance of these markers in human tissues. Fortunately, recent studies discovered a list of protein markers whose combination will enhance the diagnostic efficacy for PC. Moreover, the stability and abundance of DNA and microRNAs (miRNAs) in human tissues also shed light on their clinical utility as PC markers.

\section{Recent Progress in PC}

PC is a highly aggressive malignancy with a median survival time of less than 1 year. Clinical experience has identified its profound resistance to extant treatments. Surgical resection remains the only chance for cure of $\mathrm{PC}$ patients as comprehensive treatment showed a less satisfactory effect on PC survival [2, 3], and development of efficient early detection modality ranks the key project for oncologists. Extensive histopathological studies have evaluated pancreatic neoplasms and identified different precursor lesions as having the potential to evolve into highly malignant and invasive PC, including $\mathrm{CP}$, pancreatic intraepithelial neoplasia (PanIN), mucinous cystic neoplasms and intraductal papillary mucinous neoplasms 
(IPMNs) [4, 5]. Employing PanIN lesions, which appear to be the most common precursor pancreatic lesions, as the model to explore the transformation course of PC, a list of precancerous lesions were sequentially identified. The development course of PC is somewhat similar to that of the adenoma-carcinoma sequence in the development of colon cancer [6].

With the advancement of molecular biology, an explosion of knowledge defined well the genetic events accompanying the sequencing transformation course of PC. Combination analysis with clinicopathological parameters further characterized the clinical significance of these events in diagnosis, prognosis prediction, operation selection, etc. K-ras is the most notable and universal oncogene identified in PC cells. Although occasionally occurring in normal pancreatic tissue and detected in only $30 \%$ of pancreatic lesions at the earliest stage of histopathological disturbance [7], the frequency of K-ras activation increases as the disease progresses, and it is found in nearly all PC cases, making this activation seem to be virtually essential for PC pathogenesis [8]. Identification of K-ras mutation as the first notable genetic alteration led to a great increase in understanding of PC genetics, including inactivation of tumor-suppressive genes (e.g. p16/CDKN2A, TP53, SMAD4). One global genomic study sequenced 23,219 transcripts representing 20,661 protein-coding genes in 24 PC cases, identifying a large number of genetic alterations and a core set of 12 signaling pathways and processes that are shown to have an altered gene expression in 67-100\% of PC cases [9].

Studies have shown that PC takes 20 years to grow into a detectable tumor, and during this time course, an average of 63 genetic alterations will happen in each case [9]. Two prerequisites for PC can be deduced from these facts: first, there must be immortal pancreatic cells to accumulate these genetic events; second, there must be a harsh milieu to efficiently induce the genetic events to happen. The existence of cancer stem cells meets the first prerequisite, whereas the second prerequisite may largely be attributed to chronic inflammation surrounding pancreatic cells. Lessons from ulcerative colitis showed that the inflammation process will lead to repeated cycles of epithelial cell damage and regeneration, which will definitely increase the possibility of somatic mutations and favor tumor growth and invasion.

\section{Insights into $\mathrm{CP}$}

$\mathrm{CP}$ is a progressive inflammatory disease with irreversibly functional and morphological changes caused by various etiological factors [10]. Our recent investigation on 2,008 patients with CP showed that the incidence in China is $13 / 100,000$ and an increasing trend was obvious [11]. Since inflammation is one of the major risk factor for carcinogenesis, it was quite logical to hypothesize that CP patients were more susceptible to PC. However, it was not until 1993, with the publication of an international cohort study by Lowenfels et al. [12], that the nature of the risk was clarified. In their multicenter historical cohort study, 2,015 CP cases were enrolled and 56 cancers were identified during a mean follow-up of 7.4 years. The standardized incidence ratio was 14.4 and the risk of developing PC, 20 years after diagnosis, was as high as $4 \%$. Similar results were further confirmed regarding the increased risk in CP patients, which underlines the significance of differential diagnosis between CP and PC [13].

Hereditary pancreatitis represents the best model to delineate the causal linkage between $\mathrm{CP}$ and $\mathrm{PC}$, and epidemiological analysis showed that hereditary pancreatitis ranks the strongest known risk factor for PC [1]. By the age of 70, 40\% of patients with hereditary pancreatitis will have developed pancreatic tumors [14]. Genetic studies identified germline mutations in the PRSS1 and the CFTR gene as leading to hereditary pancreatitis. One important query was subsequently raised by oncologists: are these genes actually oncogenes for development of sporadic pancreatic carcinomas? To address this question, Hengstler et al. [15] analyzed genomic DNA in pancreatic tissue for R122H mutations in the trypsinogen gene 
from 34 patients and corresponding normal tissue from 28 of these individuals, but no mutations were found. Malats et al.s' work [16] also showed that the incidence of mutations of the CFTR gene in sporadic pancreatic carcinomas was similar to that in healthy controls. These studies suggest that PRSS1 and CFTR gene mutations are not directly associated with the development of PC. Of note, hereditary pancreatitis arises mainly during or soon after childhood, which means that patients afflicted with hereditary pancreatitis will endure chronic inflammation with onset at an early age. Though mutations in the PRSS1 and CFTR genes do not directly contribute to pancreatic tumor formation, the high-risk inflammation milieu generated by them may work.

Taking a telescope look at a PC sample slide, we will easily identify two compartments as the major components of PC, the malignant ductal cells and the surrounding stromal cells, the latter accounting for $90 \%$ of the total tumor mass [17]. Previous studies established that the stroma formation was initiated and sustained by the out-of-control inflammation, and lessons from transgenetic models indicate that inflammation induced by pancreatitis will significantly accelerate pancreatic tumor formation [18-21]. Though it is evident that $\mathrm{CP}$ is a significant risk factor in inducing $\mathrm{PC}$, the detailed mechanisms underlying the progression from $\mathrm{CP}$ to $\mathrm{PC}$ have not to be well documented yet.

\section{General Concepts Concerning CP and PC}

The concept of the strong linkage between inflammation and cancer was first raised in the 19th century by Virchow, who observed the presence of inflammation cells within neoplastic tissues [22]. Subsequent epidemiological research validated his hypothesis and revealed the causal linkage between chronic inflammation and cancer. Ulcerative colitis, which is a common chronic inflammatory disease affecting the large bowel mucosa, may set the strongest evidence to whether and how inflammation affects carcinogenesis progression. Patients with ulcerative colitis have an increased predisposition to colorectal cancer, which is in the order of 10 -fold greater than in the general Western population [23]. The inflammation-cancer connection is not restricted to a subset of tumors but may be universally identified within different cancer types, including the lung, bladder, gastrointestinal tract, skin and vulva, etc. Use of anti-inflammatory medications, e.g. aspirin, is usually associated with protection against various tumors, which to some extent mirrors that inflammation is a risk factor for certain cancer types. Though both the epidemiological results and the protection effect of anti-inflammation medication on certain tumors clearly demonstrate the strong linkage between inflammation and cancer, the detailed mechanisms underlying this connection remain to be resolved.

As has been addressed in the previous section of current review, a CP-PC association has also been established independently by different groups through various human races. Nonetheless, the CP-PC connection hold certain traits different from other cancer types. For example, COX-2 levels were significantly elevated in CP patients, and targeted therapy against COX-2 appears be a good therapeutic strategy to treat CP [24]. However, aspirin, the most commonly used COX-2 inhibitor, exhibits controversial effects on PC [25]. Extended periods of regular aspirin use might even increase the risk of PC among women [26]. These controversial results hinted that there may be a more complex network among pancreatic cells and the inflammatory milieu, and a list of comprehensive study modalities were developed to explore or validate the current knowledge framework concerning the inflammation-cancer

connection in PC. Based on this notion, multiple animal models have been established for dissecting the effects of inflammatory mediators and anti-inflammatory drugs on CP-PC progression. The currently used animal models were primarily developed based on the 
Fig. 1. Mechanisms linking $\mathrm{CP}$ and PC via intrinsic and extrinsic signaling pathways.
Kong et al.: Chronic Pancreatitis and Pancreatic Cancer

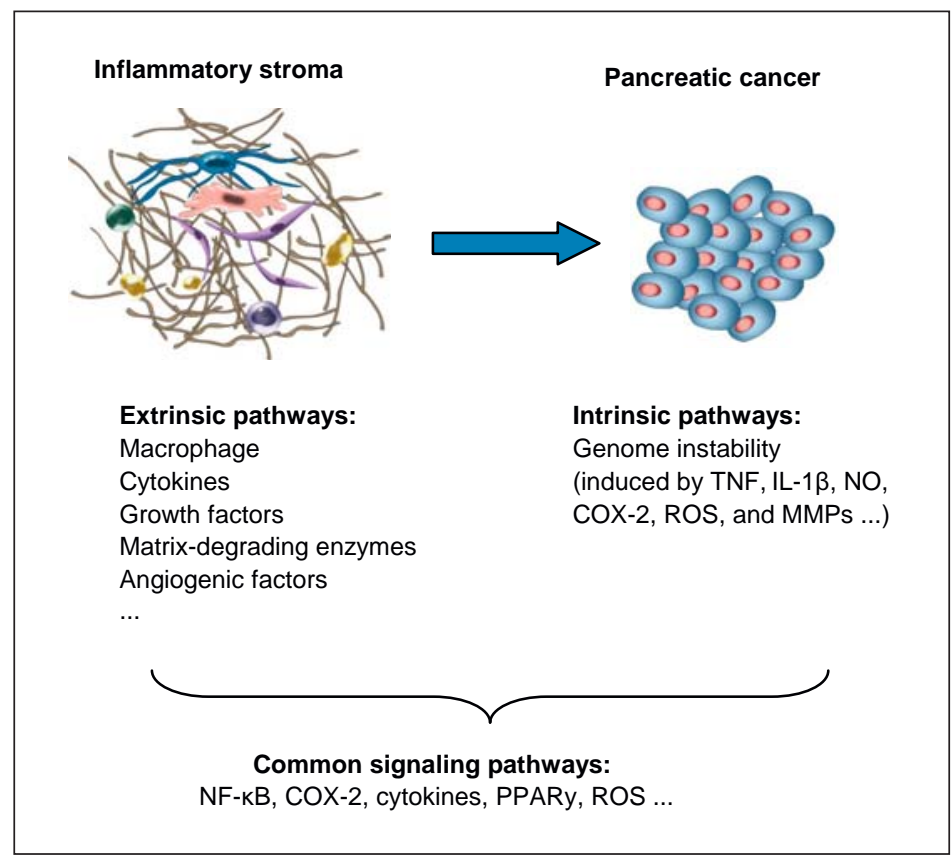

genetic activation of resident K-ras oncogenes knocked in within the endogenous K-ras locus. These models faithfully reproduce the histological lesions that characterize human pancreatic tumors, and display a desmoplastic stroma and inflammatory responses that closely resemble those observed in human patients. To be noted, these animals will not develop PC unless they undergo pancreatic damage in the form of pancreatitis. These results further established the indispensable functions of inflammation in pancreatic carcinogenesis. Employing genetic animal models, a list of mechanisms were identified linking pancreatitis and PC, which can be artificially subdivided into intrinsic and extrinsic pathways (fig. 1).

\section{Extrinsic Pathways Bridging CP and PC}

Besides neoplastic cells, the 'other half' of the PC mass is composed of a stroma constituted of fibroblasts, vessels and leukocytes. Inflammation is the primary match that sets the fire of the robust stroma reaction surrounding PC cells. Of note, inflammatory cells, especially leukocytes, are the major actors engaged in initiating and sustaining the inflammation reaction. The leukocytes within the tumor stroma, as well as the cytokines and growth factors derived from the leukocytes, constitute the complex extrinsic pathways which play a core role in the tumor formation process.

Tumor-associated macrophages (TAMs) are the principal leukocyte subset driving amplification of the inflammatory response in the tumor milieu. They primarily belong to the myeloid cell lineage and derive from myeloid progenitor cells. These precursor cells are located in the bone marrow; upon maturation, monocytes are released into the bloodstream. At the recruitment by certain chemokines, e.g. CCL2 and CCL5, TAMs accumulate in tumor stroma, where they will be taught to facilitate cancer progression. TAMs assist tumor cell malignant behavior in many ways by releasing cytokines, growth factors and matrix-degrading enzymes and a host of angiogenic factors. A list of molecular alterations are involved in the TAM-PC connection. For example, administration of agonist CD40 antibody will activate macrophages, helping macrophages infiltrate tumors became tumoricidal, and facilitate the depletion of tumor stroma [27]. TAMs may also secrete MIP3 $\alpha$ to increase the migration 
ability of PC cells by binding to the transmembrane receptor CCR6 [28]. Another work showed that TAMs could convey proangiogenetic effects to PC cells. Blocking angiopoietin-2, a TIE2 ligand and angiogenic factor, could impede the upregulation of TIE2 in TAMs and decrease tumor angiogenesis of PC [29]. Furthermore, TAMs could help maintain the cancer stem cells, which have been linked to chemoresistance, metastatic dissemination and the induction of immune suppression [30]. Blocking the connection between TAMs and cancer stem cells will help eliminate the cancer stem cells, improve chemotherapeutic response and generate promising therapeutic efficacy. In conclusion, TAMs and the complex cytokines network greatly enhance the tumorigenesis of PC. Targeted treatment against TAM may be promising in future PC therapeutics.

\section{Intrinsic Pathways within PC Cells Induced by CP}

The horrible lethality of PC may largely be attributed to its abundant genetic alterations. Previous publications identified that each PC case contains an average of 63 genetic alterations, most of which are point mutations. These alterations defined a core set of 12 cellular signaling pathways [9]. However, the rates of spontaneous mutations within normal pancreatic cells are very low. Compared with normal pancreatic cells, the widespread destabilization of gene copy number and nucleotide sequence assured us that instability of the genome is universally inherent within PC cells. In normal cells, there is an extraordinary ability of genome maintenance systems to detect and resolve defects in the DNA, whereas in cancer cells, the protection mechanisms are broken down, which enables these cells to accumulate more genetic alterations and advantageously develop into a tumor.

Previous publications established the concept that the inflammation microenvironment is involved in genome instability of PC cells. For example, Bielas et al.s' work [31] validated that the mutation rate in the inflamed microenvironment was higher than in normal tissues, with a mutation frequency of $4 \times 10^{-8}$ and $1 \times 10^{-8}$ per base pair, respectively. Another work reported that $\mathrm{p} 53$, a critical tumor suppressor engaged in protecting genomes from instability, shows high frequency of mutations in CP [32]. Higher incidence of mutations within PC cells may largely be attributed to the deregulating DNA repair systems and altered cell cycle checkpoints, whereas derivatives generated by inflammatory cells should be blamed for the destruction against these genome maintainers. For example, inflammatory cytokines, e.g. TNF and IL-1 $\beta$, could induce HIF- $\alpha$ in PC cells, which may destruct the mismatch repair mechanisms and lead to instability of genomes [33]. Nitric oxide (NO) is another important mediator derived from the inflammation microenvironment. Studies showed that NO could induce upregulation of DNA methyltransferase, which results in promoter silencing and loss of gene expression of the mismatch repair member hMLH1 [34]. NO and its derivatives could also inhibit the function of p53 and are associated with p53 mutations [35], which will significantly attenuate its detection and repair ability against genome instability. Other inflammatory elements, including COX-2, ROS, and MMPs etc., can also hamper the genome surveillance machinery. Through these different mechanisms, inflammatory elements render the cancer genomes instable, which leads to a genomically heterogeneous population of expanding cells naturally selected for their ability to proliferate, invade distant tissues and evade host defenses.

\section{Common Genetic Alterations Involved in PC and CP}

Epidemiological data identified inflammation as a significant risk factor for solid tumors. A list of studies showed that both hereditary and sporadic forms of CP are associated with increased risk of PC. In such context, recent interests of oncologists have shifted from PC cells 
to the inflammatory milieu surrounding them and identified a list of nodal targets involved in inflammation-associated carcinogenesis. Targeted therapy against certain molecules generates promising effects in the clinic. Novel therapeutics targeting the extrinsic and intrinsic pathways bridging CP-PC connections will decrease the high levels of tumorpromoting properties of the inflammatory cells and ultimately normalize the inflammatory network to regain a normal overall host response (fig. 1).

\section{Cytokines}

The progressive and irreversible desmoplasia persisting within CP is largely attributed to the cytokines existing within stroma. A large profile of cytokines has been identified within CP, including TNF- $\alpha$, IL-6, IL-8, PDGF, TGF- $\beta$, etc. Of note, the same pattern of chemokines has been found to be increased in PC [36]. As pancreatic inflammation represents an early step in the development of PC, it is quite logical to believe that these cytokines are engaged in pancreatic carcinogenesis. Numerous studies have validated the tumor-promoting roles of these cytokines in pancreatic carcinogenesis. For example, TNF- $\alpha$ is normally expressed in the context of pancreatic acinar cell injury, whereas in CP, TNF- $\alpha$ can upregulate PDGF expression, which is known to strongly stimulate fibrogenesis [37]. PDGF as well as other TNF- $\alpha$ downstream targets, e.g. EGFR and TGF- $\alpha$, are all well-known oncogenes in pancreatic carcinogenesis [38]. Through activation of NF- $\kappa \mathrm{B}, \mathrm{TNF}-\alpha$ may also inhibit apoptosis of PC cells [39]. These data suggest that abnormal cytokine expression in CP was significantly associated with pancreatic carcinogenesis. A new generation of vaccines directed against cytokine activity could be beneficial in the future treatment of cancer [40].

\section{$N F-\kappa B$}

$\mathrm{NF}-\kappa \mathrm{B}$ is an important transcription factor proved to be involved in multiple cellular activities. The functional NF- $\mathrm{BB}$ in pancreas is a p65/p50 heterodimer. Under normal conditions, NF- $\kappa \mathrm{B}$ dimers are bound to inhibitory proteins, I $\kappa \mathrm{Bs}$, which block nuclear localization sequences and thus trap the dimers within the cytoplasm where they were inactive. However, in inflammatory response, e.g. pancreatitis, I $\kappa B s$ are degraded and NF- $\kappa B$ subsequently translocates into the nucleus, where it interacts with other transcription factors and binds to its consensus sequence on promoters of target genes, such as Bcl-X and PDCD4 [41]. Our previous work showed that enhanced NF- $\mathrm{KB}$ activity was associated with increased severity of acute and chronic pancreatitis [41]. NF- $\mathrm{KB}$ activity is ubiquitously unregulated in a number of cancer types. Evidence from different groups suggests that the role that NF- $\kappa \mathrm{B}$ played in carcinogenesis may be through its inhibition of apoptosis of pre-neoplastic cells and the maintenance of a pro-neoplastic microenvironment rich in pro-inflammatory mediators [42]. Targeted therapy against NF- $\mathrm{BB}$ may induce apoptosis and increase gemcitabine effectiveness in a subset of PC cells [43]. Due to the critical role that NF- $\kappa B$ plays in linking CP and PC, restoration of its expression and function may decrease the tumor-promoting effects of inflammatory cells, with hope to orchestrate the harmonious relationship between inflammation and pancreatic cells.

\section{PPARY}

PPAR $\gamma$ is a nuclear receptor highlighted with its functions as a transcription factor that can repress inflammatory genes and orchestrate inflammation homeostasis [44]. Ligands of PPAR $\gamma$ exert optimal effects in preventing the out-of-control inflammation in various tissues. For example, different PPAR $\gamma$ agonists could reduce the severity of cerulein-induced acute pancreatitis [45, 46]. Shimizu et al.'s work [47] showed that PPAR $\gamma$ agonists could inhibit the pro-inflammatory cytokine gene expression within macrophages to prevent the development of CP. Also, PPAR $\gamma$ overexpression could inhibit pro-fibrogenic activities of immortalized rat 
pancreatic stellate cells to suppress the chronic inflammation process [48]. As CP and PC are strongly associated, different groups further evaluated PPAR $\gamma$ 's impact on PCs. Their reports showed that the PPAR $\gamma$ ligand could induce growth arrest within them [49]. A list of targets, including cyclin D1, p27KiP1 and PTEN, were implicated in its anti-tumor effects. Troglitazone is a well-known PPAR $\gamma$ ligand, and clinical studies have shown that troglitazone could significantly lower PSA levels in prostate cancer patients [50]. However, its therapeutic efficacy against PC has not been evaluated in the clinic.

ROS

ROS are generated by activated neutrophils and macrophages in the setting of inflammation. Different groups validated that ROS were implicated in the pathogenesis of acute and chronic pancreatitis, and antioxidants could be potentially effective against the development of pancreatic fibrosis in patients with CP [51]. Furthermore, in the setting of highly reactive oxygen species, repeated tissue damage and regeneration will occur in CP. Of note, genotoxic effects, including DNA strand breaks, sister chromatid exchanges and formation of adducts with DNA, will be induced by ROS in this process [52]. Repeated damage-regeneration stimulus will incidentally enforce permanent genomic alterations in pancreatic cells, which further accelerate the mutation accumulation and subsequently the carcinogenesis process. Though no clinical trials have evaluated the anti-tumor effects of those compounds with antioxidant activity, epidemiological studies showed that intake of fresh fruit and vegetables appears to be inversely correlated with PC [53]. Eradication of ROS from the chronic inflammatory milieu will protect pancreatic cell DNA from genotoxic damage and perhaps lower the incidence of transformation of these cells.

\section{Differential Diagnosis between CP and PC}

$\mathrm{CP}$ and $\mathrm{PC}$ are two distinct diseases with entirely different prognoses. However, in certain cases, there is a great challenge to discriminate among them. First, just like CP tissue which is mostly made of fibrosis, more than $90 \%$ of pancreatic tumor mass is also made of the stroma element. Applying fine needle aspiration, the most commonly used technique to sample a pancreatic mass, it is hard to get the tissue containing pancreatic cells. Second, there are too many common dysregulated pathways among CP and PC tissues, such as ROS, Hedgehog, NF- $\kappa B$, etc. Until now, none of the global proteomic studies have identified cancerspecific proteins. Third, as CP patients hold more susceptibility to develop into PC, they are frequently followed up at indicated time points. However, even using the most advanced techniques, we cannot deny the existence of malignant cells hidden within a chronic inflammatory mass.

It is generally accepted that stroma formation is a critical hallmark characteristic for PC, and the transformation of pancreatic cells usually occurs in inflamed tissues. Most previous cancer biomarker studies using modern technologies are methodologically flawed as they compare samples from cancer patients with those of healthy, inflammation-free people. An accurate and non-invasive test to differentiate PC from CP would be extremely helpful to detect PC at an early stage and help physicians make the right treatment decisions, which will increase patients' survival.

\section{Protein Biomarkers}

CA19-9 is the most widely utilized tumor maker for PC, and its sensitivity is $~ 80 \%$; this value is considerably diminished $(\sim 55 \%)$ in small, resectable cancers $(<3 \mathrm{~cm})$. Furthermore, in high-risk, asymptomatic individuals harboring IPMNs or high-grade PanINs, serum 
CA19-9 is often normal. CP tissues also hold a high positive rate of CA19-9 expression [54]. These data show that CA19-9 is not a perfect marker to detect those non-malignant pancreatic tumors, nor is it suitable to distinguish PC from CP and other non-neoplastic pancreatic diseases. Recent efforts have been devoted to markers with a capacity to distinguish PC from $\mathrm{CP}$ cases and have identified a list of markers with potential clinical utility. For example, employing quantitative RT-PCR and immunohistochemistry, one work showed that expression analysis of UHRF1, ATP7A and aldehyde oxidase 1 in combination could potentially provide a useful additional diagnostic tool for fine needle-aspirated or cytological specimens obtained during endoscopic investigations [55]. PAM4 is a monoclonal antibody expressed by $90 \%$ of pancreatic ductal adenocarcinomas as well as the precursor lesions PanIN and IPMN, and shows high specificity for pancreatic ductal adenocarcinoma and precursor lesions versus benign, non-neoplastic pancreatic tissues. Furthermore, approximately $80 \%$ of CP patients are negative for circulating PAM4 antigen, which highlights PAM4's potential use in the differential diagnosis between CP and PC [54].

\section{DNA and RNA Biomarkers}

Previous notions believed that nuclease in human plasma was too rich that DNA and RNA fragments could not be stably detected as markers for cancer detection. However, recent methodological studies showed that DNA and RNA molecules could also serve as markers with potential clinical utility. For example, KRAS2 mutations in codon 12 could stably be detected in circulating deoxyribonucleic acid, and its positive rate is significantly higher in PC than in CP patients (47 vs. 13\%). Combined normal serum CA19-9 and absence of circulating KRAS2 mutations may significantly increase the differential diagnosis efficacy between CP and PC [56]. Our study also showed that the detection of KRAS gene mutations (codons 12 and 13) in samples of endoscopic ultrasound-guided fine needle aspiration biopsy was feasible for the diagnosis of PC [57].

miRNAs are endogenous, small, non-coding RNAs that repress the expression of target mRNAs. Our recent works identified that miRNAs can be stably detected in plasma and may serve as good markers for PC diagnosis [58-60]. Among 7 miRNAs evaluated in our study, miR-21 was able to distinguish PC from CP and healthy patients, whereas miR-155 and miR-196a were able to differentiate sera from patients with sick pancreas (PC/CP) from those of patients with normal pancreas. Another group from Denmark further quantitated miRNAs in whole blood from PC and CP patients as well as healthy controls. Their work identified two panels of miRNAs with potential to distinguish PC patients from CP patients and healthy controls [61].

\section{Summary}

$\mathrm{CP}$ is a common chronic inflammatory disease of unknown etiology which affects the parenchyma and functions of human pancreas. Epidemiological studies have identified that $\mathrm{CP}$ is a major risk factor for $\mathrm{PC}$, whereas numerous extrinsic and intrinsic pathways have been identified to be involved in the connection of CP and PC. Of note, a list of genetic alterations were commonly detected in CP and PC tissues, and targeted therapy against certain alterations exhibits optimal efficacy in PC treatment. As CP and PC patients hold entirely distinct prognoses, the differential diagnosis between the two diseases is extremely important in the clinic. Most cancer biomarker studies using modern technologies are methodologically flawed as they compare samples from cancer patients with those of healthy, inflammation-free people. Future studies regarding PC markers should exclude molecules that are dysregulated in CP tissues. 


\section{Acknowledgement}

The work was supported by Shanghai Science and Technology Key Project No. 11JC1416402, Changhai Hospital '1255' Project No. CH125510308 (to Y. Du) and Shanghai Yang Fan Project No. 14YF1405600 (to X. Kong).

\section{Author Contributions}

X. Kong wrote the manuscript; Y. Du and Z. Li designed the theme and framework; T. Sun and F. Kong searched the literature and collected the references.

\section{Disclosure Statement}

The authors declare that there is no conflict of interests regarding the publication of this review.

\section{References}

1 Schneider A, Whitcomb DC: Hereditary pancreatitis: a model for inflammatory diseases of the pancreas. Best Pract Res Clin Gastroenterol 2002;16:347-363.

-2 Du YQ, Li ZS, Jin ZD: Endoscope-assisted brachytherapy for pancreatic cancer: from tumor killing to pain relief and drainage. J Interv Gastroenterol 2011;1:23-27.

-3 Du Y, Jin Z, Meng H, et al: Long-term effect of gemcitabine-combined endoscopic ultrasonography-guided brachytherapy in pancreatic cancer. J Interv Gastroenterol 2013;3:18-24.

4 Brugge WR, Lauwers GY, Sahani D, et al: Cystic neoplasms of the pancreas. N Engl J Med 2004;351:1218-1226.

5 Maitra A, Fukushima N, Takaori K, et al: Precursors to invasive pancreatic cancer. Adv Anat Pathol 2005;12: 81-91.

6 Vogelstein B, Fearon ER, Hamilton SR, et al: Genetic alterations during colorectal-tumor development. N Engl J Med 1988;319:525-532.

7 Klimstra DS, Longnecker DS: K-ras mutations in pancreatic ductal proliferative lesions. Am J Pathol 1994;145: 1547-1550.

8 Rozenblum E, Schutte M, Goggins M, et al: Tumor-suppressive pathways in pancreatic carcinoma. Cancer Res 1997;57:1731-1734.

-9 Jones S, Zhang X, Parsons DW, et al: Core signaling pathways in human pancreatic cancers revealed by global genomic analyses. Science 2008;321:1801-1806.

10 Liao Z, Jin G, Cai D, et al: Guidelines: diagnosis and therapy for chronic pancreatitis. J Interv Gastroenterol 2013;3:133-136.

11 Wang LW, Li ZS, Li SD, et al: Prevalence and clinical features of chronic pancreatitis in China: a retrospective multicenter analysis over 10 years. Pancreas 2009;38:248-254.

12 Lowenfels AB, Maisonneuve P, Cavallini G, et al: Pancreatitis and the risk of pancreatic cancer. International Pancreatitis Study Group. N Engl J Med 1993;328:1433-1437.

13 Bansal P, Sonnenberg A: Pancreatitis is a risk factor for pancreatic cancer. Gastroenterology 1995;109:247251.

14 Lowenfels AB, Maisonneuve P, DiMagno EP, et al: Hereditary pancreatitis and the risk of pancreatic cancer. International Hereditary Pancreatitis Study Group. J Natl Cancer Inst 1997;89:442-446.

15 Hengstler JG, Bauer A, Wolf HK, et al: Mutation analysis of the cationic trypsinogen gene in patients with pancreatic cancer. Anticancer Res 2000;20:2967-2974.

16 Malats N, Casals T, Porta M, et al: Cystic fibrosis transmembrane regulator (CFTR) DeltaF508 mutation and 5T allele in patients with chronic pancreatitis and exocrine pancreatic cancer. PANKRAS II Study Group. Gut 2001;48:70-74.

17 Neesse A, Michl P, Frese KK, et al: Stromal biology and therapy in pancreatic cancer. Gut 2011;60:861-868.

18 Carrière C, Young AL, Gunn JR, et al: Acute pancreatitis markedly accelerates pancreatic cancer progression in mice expressing oncogenic Kras. Biochem Biophys Res Commun 2009;382:561-565.

19 Gidekel Friedlander SY, Chu GC, Snyder EL, et al: Context-dependent transformation of adult pancreatic cells by oncogenic K-Ras. Cancer Cell 2009;16:379-389.

20 Guerra C, Schuhmacher AJ, Cañamero M, et al: Chronic pancreatitis is essential for induction of pancreatic ductal adenocarcinoma by K-Ras oncogenes in adult mice. Cancer Cell 2007;11:291-302.

21 Morris JP 4th, Cano DA, Sekine S, et al: Beta-catenin blocks Kras-dependent reprogramming of acini into pancreatic cancer precursor lesions in mice. J Clin Invest 2010;120:508-520. 
22 2008;22:65-73.

Pan X, Arumugam T, Yamamoto T, et al: Nuclear factor-kappaB p65/relA silencing induces apoptosis and 8151.

44 Glass CK, Saijo K: Nuclear receptor transrepression pathways that regulate inflammation in macrophages and T cells. Nat Rev Immunol 2010;10:365-376.

45 Hashimoto K, Ethridge RT, Saito H, et al: The PPARgamma ligand, 15d-PGJ2, attenuates the severity of ceruleininduced acute pancreatitis. Pancreas 2003;27:58-66.

46 Cuzzocrea S, Pisano B, Dugo L, et al: Rosiglitazone, a ligand of the peroxisome proliferator-activated receptorgamma, reduces acute pancreatitis induced by cerulein. Intensive Care Med 2004;30:951-956.

47 Shimizu K, Shiratori K, Hayashi N, et al: Thiazolidinedione derivatives as novel therapeutic agents to prevent the development of chronic pancreatitis. Pancreas 2002;24:184-190.

48 Jaster R, Lichte P, Fitzner B, et al: Peroxisome proliferator-activated receptor gamma overexpression inhibits pro-fibrogenic activities of immortalised rat pancreatic stellate cells. J Cell Mol Med 2005;9:670-682.

49 Elnemr A, Ohta T, Iwata K, et al: PPARgamma ligand (thiazolidinedione) induces growth arrest and differentiation markers of human pancreatic cancer cells. Int J Oncol 2000;17:1157-1164.

-50 Mueller E, Smith M, Sarraf P, et al: Effects of ligand activation of peroxisome proliferator-activated receptor gamma in human prostate cancer. Proc Natl Acad Sci USA 2000;97:10990-10995.

-51 Asaumi H, Watanabe S, Taguchi M, et al: Externally applied pressure activates pancreatic stellate cells through the generation of intracellular reactive oxygen species. Am J Physiol Gastrointest Liver Physiol 2007;293: G972-G978. 
52 Jackson AL, Loeb LA: The contribution of endogenous sources of DNA damage to the multiple mutations in cancer. Mutat Res 2001;477:7-21.

53 Wiseman H, Halliwell B: Damage to DNA by reactive oxygen and nitrogen species: role in inflammatory disease and progression to cancer. Biochem J 1996;313:17-29.

54 Shi C, Merchant N, Newsome G, et al: Differentiation of pancreatic ductal adenocarcinoma from chronic pancreatitis by PAM4 immunohistochemistry. Arch Pathol Lab Med 2014;138:220-228.

55 Crnogorac-Jurcevic T, Gangeswaran R, Bhakta V, et al: Proteomic analysis of chronic pancreatitis and pancreatic adenocarcinoma. Gastroenterology 2005;129:1454-1463.

56 Maire F, Micard S, Hammel P, et al: Differential diagnosis between chronic pancreatitis and pancreatic cancer: value of the detection of KRAS2 mutations in circulating DNA. Br J Cancer 2002;87:551-554.

57 Wang X, Gao J, Ren Y, et al: Detection of KRAS gene mutations in endoscopic ultrasound-guided fine-needle aspiration biopsy for improving pancreatic cancer diagnosis. Am J Gastroenterol 2011;106:2104-2111.

58 Kong X, Du Y, Wang G, et al: Detection of differentially expressed microRNAs in serum of pancreatic ductal adenocarcinoma patients: miR-196a could be a potential marker for poor prognosis. Dig Dis Sci 2011;56: 602-609.

59 Liu J, Gao J, Du Y, et al: Combination of plasma microRNAs with serum CA19-9 for early detection of pancreatic cancer. Int J Cancer 2012;131:683-691.

60 Liu R, Chen X, Du Y, et al: Serum microRNA expression profile as a biomarker in the diagnosis and prognosis of pancreatic cancer. Clin Chem 2012;58:610-618.

61 Schultz NA, Dehlendorff C, Jensen BV, et al: MicroRNA biomarkers in whole blood for detection of pancreatic cancer. JAMA 2014;311:392-404. 